J. Appl. Numer. Optim. 2 (2020), No. 3, pp. 353-371

Available online at http://jano.biemdas.com

https://doi.org/10.23952/jano.2.2020.3.06

\title{
THE S-ITERATIVE TECHNIQUES ON HADAMARD MANIFOLDS AND APPLICATIONS
}

\author{
D.R. SAHU ${ }^{1, *}$, FEEROZ BABU ${ }^{2}$, SHIKHER SHARMA ${ }^{1}$ \\ ${ }^{1}$ Department of Mathematics, Banaras Hindu University, Varanasi, India \\ ${ }^{2}$ Department of Mathematics, I.K. Gujral Punjab Technical University, Kapurthala, India
}

\begin{abstract}
In this paper, we develop S-iterative techniques in terms of exponential mappings for finding fixed points of nonexpansive mappings on Hadamard manifolds. To demonstrate numerical applicability, we show our proposed methods are faster than the existing results in the literature. For the applications, we focus on the common singularities of set-valued monotone vector fields and convex feasibility problems.
\end{abstract}

Keywords. Nonexpansive mappings; S-iteration method; Monotone vector fields; Inclusion problems; convex feasibility problems.

\section{INTRODUCTION}

Let $K$ be a nonempty closed convex subset of a Banach space $E$ and $T: K \rightarrow K$ be an operator. Many nonlinear problems of applied mathematics can be formulated as the following equation, which is known as a fixed point problem

$$
x=T x, \quad \text { for some } x \in K .
$$

The solution set of fixed point problem (1.1) is denoted as $\operatorname{Fix}(T)$. To study the asymptotic behavior of operator $T$ in the fixed point theory, many researchers have a keen interest to develop algorithms for finding the approximate solutions of problem (1.1). For example, if $T$ is a contraction map, then there is a well known Picard's iteration $\left\{T^{n} x\right\}$, which converges strongly to the solution of problem (1.1). If $T$ is a nonexpansive map, then Picard's iterates may fail to converge. In fact, this drawback is the key motivation to construct new types of algorithms for problem (1.1), for instance, Mann's iteration, Halpern's iteration, S-iteration and etc., see $[1,3,4,5,9,10,11,13,17]$. From now on, we denote $\mathbb{N}_{0}$ by $\mathbb{N} \cup\{0\}$. Mann [9] introduced the following algorithm, which has been extensively studied $([3,12,16])$,

$$
x_{0} \in K, \quad x_{n+1}=\left(1-\alpha_{n}\right) x_{n}+\alpha_{n} T x_{n}, \quad \forall n \in \mathbb{N}_{0},
$$

\footnotetext{
${ }^{*}$ Corresponding author.

E-mail addresses: drsahudr@gmail.com (D.R. Sahu), firoz77b@gmail.com (F. Babu), shikhers043@gmail.com (S. Sharma).

Received May 28, 2020; Accepted October 15, 2020.

(C)2020 Journal of Applied and Numerical Optimization
} 
where $\left\{\alpha_{n}\right\}$ is the sequence of scalar in $(0,1)$. However, Mann's algorithm (1.2) converges weakly. Further, Halpern [11] defined the following algorithm, which converges strongly to a solution of problem (1.1).

On the other hand, in 2007, Agarwal, O'Regan and Sahu [2] introduced the S-iteration method in a Banach space $E$ as follows:

$$
\left\{\begin{array}{l}
x_{n+1}=\left(1-\alpha_{n}\right) T x_{n}+\alpha_{n} T y_{n}, \\
y_{n}=\left(1-\beta_{n}\right) x_{n}+\beta_{n} T x_{n}, \quad \forall n \in \mathbb{N}_{0},
\end{array}\right.
$$

where $\left\{\alpha_{n}\right\}$ and $\left\{\beta_{n}\right\}$ are the sequences of scalars in $(0,1)$. In the recent years, the S-iterative technique and its variants have attracted many authors as an alternative iteration method for fixed-point problems, common fixed point problems and real world problems; see, e.g., [17, 18, 19] and the references therein. In 2011, Sahu [20] introduced the normal S-iteration in the setting of Banach space $E$ as follows:

$$
\left\{\begin{array}{l}
x_{n+1}=T y_{n}, \\
y_{n}=\left(1-\alpha_{n}\right) x_{n}+\alpha_{n} T x_{n}, \quad \forall n \in \mathbb{N}_{0},
\end{array}\right.
$$

where $\left\{\alpha_{n}\right\}$ is the sequence of scalar in $(0,1)$. These iteration methods (1.3) and (1.4) have better rate of convergence than the Mann iteration method for finding an approximate solution of problem (1.1). For more details, we refer to [1, 2, 17, 20].

Recently, the following S-iterative algorithms were studied by Sahu, Pitea and Verma [17] in terms of nonexpansive mappings $T, S: K \rightarrow K$ :

Algorithm 1.1. For arbitrary $x_{0} \in K$, define a sequence $\left\{x_{n}\right\}$ in $K$ by

$$
\left\{\begin{array}{l}
x_{n+1}=\left(1-\alpha_{n}\right) S x_{n}+\alpha_{n} T y_{n}, \\
y_{n}=\left(1-\beta_{n}\right) x_{n}+\beta_{n} T x_{n}, \quad \forall n \in \mathbb{N}_{0},
\end{array}\right.
$$

where $\left\{\alpha_{n}\right\}$ and $\left\{\beta_{n}\right\}$ are sequences of scalars in $(0,1)$.

Algorithm 1.2. For arbitrary $x_{0} \in K$, define a sequence $\left\{x_{n}\right\}$ in $K$ by

$$
\left\{\begin{array}{l}
x_{n+1}=\left(1-\alpha_{n}\right) S x_{n}+\alpha_{n} T y_{n}, \\
y_{n}=\left(1-\beta_{n}\right) S x_{n}+\beta_{n} T z_{n}, \\
z_{n}=\left(1-\delta_{n}\right) x_{n}+\delta_{n} T x_{n}, \quad \forall n \in \mathbb{N}_{0}
\end{array}\right.
$$

where $\left\{\alpha_{n}\right\},\left\{\beta_{n}\right\}$ and $\left\{\delta_{n}\right\}$ are sequences of scalars in $(0,1)$.

Let $A, B: E \rightrightarrows E$ be set-valued operators. Consider the following inclusion problem:

Find $\bar{x} \in E$ such that $\mathbf{0} \in A \bar{x}, \cap B \bar{x}$.

where $\mathbf{0}$ is a zero vector in $E$. For finding solutions of problem (1.5), Sahu, Pitea and Verma [17] developed a new proximal point algorithms on the basis of Algorithms 1.1 and 1.2.

Recently, a large number of extensions and modifications in the past two decades have been established in different fields of applied mathematics to approximate the solution of problem (1.1). In particular, Li, López and Martín-Márquez [13] extended Mann's and Halpern's iterative methods on Hadamard manifolds. 
Motivated by the wide range applications of the S-iterative technique, the purpose of this paper to develop the S-iterative methods in the setting of Hadamard manifolds. Our proposed method converges faster than the one in [13] with mild assumptions on the scalars. We study the $\mathrm{S}$-iteration techniques in terms of exponential mappings on setting of Hadamard manifolds and give some novel applications to the common solutions of inclusion problem (1.5) and convex feasibility problems.

This paper consists of five sections. In Section 2, we gather all the concepts from the Riemannian manifolds. Section 3 deals with the constructions of the algorithms and their convergence under the bounded and unbounded assumptions on the scalars. In Section 4, we illustrate a numerical example that shows our proposed methods are faster than previous methods. The last section is devoted to the applications of the inclusion problems and convex feasibility problems.

\section{PRELIMINARIES}

In this section, we recall some fundamental definitions, properties and notations of Riemannian manifolds. For more intricate details, we refer to [8, 21, 22].

Let $\mathbb{M}$ be a $m$-dimensional differentiable manifold and $p \in \mathbb{M}$. The set of all tangents at point $p$ is called the tangent space of $\mathbb{M}$ at $p \in \mathbb{M}$, denoted by $T_{p} \mathbb{M}$, which forms a vector space of dimension $m$. Tangent bundle of $\mathbb{M}$ is defined as $T \mathbb{M}=\bigcup_{p \in \mathbb{M}} T_{p} \mathbb{M}$.

A Riemannian metric $\langle\cdot, \cdot\rangle_{p}$ on $T_{p} \mathbb{M}$ is an inner product on $T_{p} \mathbb{M}$. If $\langle\cdot, \cdot\rangle_{p}$ is a Riemannian metric on $T_{p} \mathbb{M}$ for every $p \in \mathbb{M}$, then the smooth mapping $\langle\cdot, \cdot\rangle: p \longmapsto\langle\cdot, \cdot\rangle_{p}$ is called a Riemannian metric on $\mathbb{M}$. The corresponding norm to inner product $\langle\cdot, \cdot\rangle_{p}$ on $T_{p} \mathbb{M}$ is denoted by $\|\cdot\|_{p}$. We omit the subscript $p$ if there is no confusion occurs. We always assume that $\mathbb{M}$ can be endowed with a Riemannian metric $\langle\cdot, \cdot\rangle$. A differentiable manifold $\mathbb{M}$ with the Riemannian metric $\langle\cdot, \cdot\rangle$ is said to be a Riemannian manifold.

Let $p, q \in \mathbb{M}$ and $\gamma:[a, b] \rightarrow \mathbb{M}$ be a piecewise smooth curve joining $p$ to $q$. Then, the length of the curve $\gamma$ is defined as

$$
L(\gamma)=\int_{a}^{b}\left\|\gamma^{\prime}(t)\right\| d t
$$

where $\gamma^{\prime}(t) \in T_{\gamma(t)} \mathbb{M}$ is tangent vector at the point $\gamma(t) \in \mathbb{M}$. The minimal length of all curves joining $p$ to $q$ is called the Riemannian distance and is denoted by $d(p, q)$.

A single-valued vector field on a differentiable manifold $\mathbb{M}$ is a smooth mapping $X: \mathbb{M} \rightarrow$ $T \mathbb{M}$ such that, for every $p \in \mathbb{M}$, there exists a tangent vector $X(p) \in T_{p} \mathbb{M}$. Let $\Delta$ be the LeviCivita connection associated with the Riemannian manifold $M$. A smooth vector field $X$ along $\gamma$ is said to be parallel if $\Delta_{\gamma^{\prime}} X=0$. If $\gamma^{\prime}$ is parallel along $\gamma$, i.e., $\Delta_{\gamma^{\prime}} \gamma^{\prime}=0$, then $\gamma$ is said to be geodesic. In this case, $\left\|\gamma^{\prime}\right\|$ is a constant. Moreover, if $\left\|\gamma^{\prime}\right\|=1$, then $\gamma$ is called a normalized geodesic. A geodesic joining $p$ to $q$ in the Riemannian manifold $\mathbb{M}$ is said to be a minimal geodesic if its length is equal to $d(p, q)$.

A Riemannian manifold $\mathbb{M}$ is said to be complete if for any $p \in \mathbb{M}$ all geodesic emanating from $p$ are defined for all $t \in \mathbb{R}$. By Hopf-Rinow Theorem [21], if $\mathbb{M}$ is complete, then any pair of points in $\mathbb{M}$ can be joined by a minimal geodesic. Moreover, $(\mathbb{M}, d)$ is a complete metric space. If $\mathbb{M}$ is a complete Riemannian manifold, then the exponential map $\exp _{p}: T_{p} \mathbb{M} \rightarrow \mathbb{M}$ at $p \in \mathbb{M}$ is defined by

$$
\exp _{p} v=\gamma_{v}(1 ; p), \quad \forall v \in T_{p} \mathbb{M}
$$


where $\gamma_{v}(. ; p): \mathbb{R} \rightarrow \mathbb{M}$ is a unique geodesic starting from $p$ with velocity $v$, i.e., $\gamma_{v}(0 ; p)=p$ and $\gamma_{v}^{\prime}(0 ; p)=v$. It is known that $\exp _{p}(t v)=\gamma_{v}(t ; p)$ for each real number $t$ and $\exp _{p} \mathbf{0}=\gamma_{\mathbf{0}}(0 ; p)=$ $p$, where $\mathbf{0}$ is the zero tangent vector. Note that the exponential map $\exp _{p}$ is diffeomorphism on $T_{p} \mathbb{M}$. Moreover, for any $p, q \in \mathbb{M}$, we have $d(p, q)=\left\|\exp _{p}^{-1} q\right\|$.

A complete simply connected Riemannian manifold of nonpositive sectional curvature is called a Hadamard manifold. Throughout the remainder of the paper, we will always assume that $\mathbb{M}$ is a $m$-dimensional Hadamard manifold.

Proposition 2.1. [21] Let $\mathbb{M}$ be an m-dimensional Hadamard manifold and $p \in \mathbb{M}$. Then $\exp _{p}: T_{p} \mathbb{M} \rightarrow \mathbb{M}$ is a diffeomorphism and, for any two points $p, q \in \mathbb{M}$, there exists a unique normalized geodesic joining $p$ to $q$, which is in fact a minimal geodesic. Let $\gamma:[0,1] \rightarrow \mathbb{M}$ be the desired geodesic i.e., $p=\gamma(0)$ and $q=\gamma(1)$. Then

$$
\gamma(t)=\exp _{p}\left(t \exp _{p}^{-1} q\right), \quad \forall t \in[0,1] .
$$

A subset $K$ of a Riemannian manifold $\mathbb{M}$ is said to be a geodesic convex if, for any two points $p$ and $q$ in $K$, any geodesic joining $p$ to $q$ is contained in $K$, that is, for all $a, b \in \mathbb{R}$ such that $p=\gamma(a)$ and $q=\gamma(b), \gamma(a t+(1-t) b) \in K$ for all $t \in[0,1]$.

From now on, $K \subset \mathbb{M}$ will denote a nonempty, closed and geodesic convex subset of a Hadamard manifold $\mathbb{M}$. The projection map onto $K$ is given by

$$
P_{K}(q)=\{r \in K: d(q, r) \leq d(q, p), \forall p \in K\}, \forall q \in \mathbb{M} .
$$

A function $f: \mathbb{M} \rightarrow \mathbb{R}$ is said to be a geodesic convex if, for any geodesic $\gamma:[a, b] \rightarrow \mathbb{M}$, the composition function $f \circ \gamma:[a, b] \rightarrow \mathbb{R}$ is convex, that is,

$$
(f \circ \gamma)(a t+(1-t) b) \leq t(f \circ \gamma)(a)+(1-t)(f \circ \gamma)(b), \quad \forall t \in[0,1] \text { and } a, b \in \mathbb{R} \text {. }
$$

Proposition 2.2. [21] Let $d: \mathbb{M} \times \mathbb{M} \rightarrow \mathbb{R}$ be a metric function. Then $d$ is a convex function with respect to product Riemannian metric, i.e., given any pair of geodesics $\gamma_{1}:[0,1] \rightarrow \mathbb{M}$ and $\gamma_{2}:[0,1] \rightarrow \mathbb{M}$, the following inequality holds:

$$
d\left(\gamma_{1}(t), \gamma_{2}(t)\right) \leq(1-t) d\left(\gamma_{1}(0), \gamma_{2}(0)\right)+t d\left(\gamma_{1}(1), \gamma_{2}(1)\right), \quad \forall t \in[0,1] .
$$

In particular, for each $p \in \mathbb{M}$, the function $d(\cdot, p): \mathbb{M} \rightarrow \mathbb{R}$ is a geodesic convex function.

We mention some geometric properties from the finite dimensional Hadamard manifold $\mathbb{M}$, which are similar to the setting of Euclidean space $\mathbb{R}^{m}$.

A geodesic triangle $\Delta\left(p_{1}, p_{2}, p_{3}\right)$ in a Riemannian manifold is a set, consisting of three points $p_{1}, p_{2}, p_{3}$ and three minimal geodesics $\gamma_{1}$ : joining $p_{1}$ to $p_{2}, \gamma_{2}$ : joining $p_{2}$ to $p_{3}$ and $\gamma_{3}$ : joining $p_{3}$ to $p_{1}$.

Proposition 2.3. [14] (Comparison Theorem for Triangles) Let $\Delta\left(p_{1}, p_{2}, p_{3}\right)$ be a geodesic triangle. Denote, for each $i=1,2,3(\bmod 3)$ by $\gamma_{i}:\left[0, l_{i}\right] \rightarrow \mathbb{M}$ the geodesic joining $p_{i}$ to $p_{i+1}$. Let $l_{i}=L\left(\gamma_{i}\right)$ and $\alpha_{i}$ be the angle between tangent vectors $\gamma_{i}^{\prime}(0)$ and $\gamma_{i-1}^{\prime}\left(l_{i-1}\right)$. Then

$$
\begin{gathered}
\alpha_{1}+\alpha_{2}+\alpha_{3} \leq \pi \\
l_{i}^{2}+l_{i+1}^{2}-2 l_{i} l_{i+1} \cos \alpha_{i+1} \leq l_{i-1}^{2} .
\end{gathered}
$$

Since

$$
\left\langle\exp _{p_{i+1}}^{-1} p_{i}, \exp _{p_{i+1}}^{-1} p_{i+2}\right\rangle=d\left(p_{i}, p_{i+1}\right) d\left(p_{i+1}, p_{i+2}\right) \cos \alpha_{i+1}
$$


therefore, in terms of distance and the exponential map, inequality (2.4) can be written as

$$
d^{2}\left(p_{i}, p_{i+1}\right)+d^{2}\left(p_{i+1}, p_{i+2}\right)-2\left\langle\exp _{p_{i+1}}^{-1} p_{i}, \exp _{p_{i+1}}^{-1} p_{i+2}\right\rangle \leq d^{2}\left(p_{i-1}, p_{i}\right) .
$$

Lemma 2.1. [13] Let $\Delta(p, q, r)$ be a geodesic triangle in a Hadamard manifold $\mathbb{M}$. Then there exists $p^{\prime}, q^{\prime}, r^{\prime} \in \mathbb{R}^{2}$ such that

$$
d(p, q)=\left\|p^{\prime}-q^{\prime}\right\|, d(q, r)=\left\|q^{\prime}-r^{\prime}\right\| \text { and } d(r, p)=\left\|r^{\prime}-p^{\prime}\right\| .
$$

The triangle $\Delta\left(p^{\prime}, q^{\prime}, r^{\prime}\right)$ is called the comparison triangle of the geodesic triangle $\Delta(p, q, r)$, which is unique upto isometry of $\mathbb{M}$.

Lemma 2.2. [13] Let $\Delta(p, q, r)$ be a geodesic triangle in a Hadamard manifold $\mathbb{M}$ and $\Delta\left(p^{\prime}, q^{\prime}, r^{\prime}\right)$ be its comparison triangle.

(a) Let $\alpha, \beta, \gamma\left(\right.$ resp. $\left.\alpha^{\prime} . \beta^{\prime}, \gamma^{\prime}\right)$ be the angles of $\Delta(p, q, r)\left(\right.$ resp. $\left.\Delta\left(p^{\prime}, q^{\prime}, r^{\prime}\right)\right)$ at the vertices $p, q, r\left(\right.$ resp. $\left.p^{\prime}, q^{\prime}, r^{\prime}\right)$. Then the following inequalities holds:

$$
\alpha^{\prime} \geq \alpha, \beta^{\prime} \geq \beta \text { and } \gamma^{\prime} \geq \gamma
$$

(b) Let $z$ be a point in the geodesic joining $p$ to $q$ and $z^{\prime}$ its comparison point in the interval $\left[p^{\prime}, q^{\prime}\right]$. Suppose that $d(z, p)=\left\|z^{\prime}, p^{\prime}\right\|$ and $d(z, q)=\left\|z^{\prime}-q^{\prime}\right\|$. Then the following inequalities holds:

$$
d(z, r) \leq\left\|z^{\prime}-r^{\prime}\right\|
$$

Let $\mathscr{X}(\mathbb{M})$ be the set of all set-valued vector fields $V: \mathbb{M} \rightarrow T \mathbb{M}$ such that $V(p) \subset T_{p} \mathbb{M}$ for each $p \in \mathbb{M}$. The domain $D(V)$ of $V$ is defined by $D(V)=\{p \in \mathbb{M}: V(p) \neq \emptyset\}$.

Definition 2.1. [14] A set-valued vector field $V \in \mathscr{X}(\mathbb{M})$ is said to be

(a) monotone if, for any $x, y \in D(V)$, the following condition holds:

$$
\left\langle u, \exp _{x}^{-1} y\right\rangle \leq\left\langle v,-\exp _{y}^{-1} x\right\rangle, \quad \forall u \in V(x) \text { and } \forall v \in V(y)
$$

(b) strictly monotone if, for any $x, y \in D(V)$, the following condition holds:

$$
\left\langle u, \exp _{x}^{-1} y\right\rangle<\left\langle v,-\exp _{y}^{-1} x\right\rangle, \quad \forall u \in V(x) \text { and } \forall v \in V(y) \text {; }
$$

(c) strongly monotone if there exists $\rho>0$ such that, for any $x, y \in D(V)$, the following condition holds:

$$
\left\langle u, \exp _{x}^{-1} y\right\rangle-\left\langle v,-\exp _{y}^{-1} x\right\rangle \leq-\rho d^{2}(x, y), \quad \forall u \in V(x) \text { and } \forall v \in V(y) .
$$

Definition 2.2. [15] For a given $\mu>0$ and a set-valued vector field $V \in \mathscr{X}(\mathbb{M})$, the resolvent of the vector field $V$ of order $\mu$ is a set-valued map $J_{\mu}^{V}: \mathbb{M} \rightrightarrows T \mathbb{M}$ defined by

$$
J_{\mu}^{V}(x):=\left\{z \in \mathbb{M}: \exp _{z}^{-1} x \in \mu V(z)\right\}, \forall x \in \mathbb{M} .
$$

Definition 2.3. [15] A mapping $T: C \subset \mathbb{M} \rightarrow \mathbb{M}$ is said to be firmly nonexpansive if, for any $x, y \in C$, the function $\phi:[0,1] \rightarrow[0, \infty)$ defined by

$$
\phi(t)=d\left(\exp _{x} t \exp _{x}^{-1} T x, \exp _{y} t \exp _{y}^{-1} T y\right), \forall t \in[0,1],
$$

is nonincreasing. Here $d: \mathbb{M} \times \mathbb{M} \rightarrow \mathbb{R}^{+}$is Riemannian distance function.

Lemma 2.3. [15] A set-valued vector field $V \in \mathscr{X}(\mathbb{M})$ is monotone if and only if $J_{\mu}^{V}(x)$ is single valued and firmly nonexpansive for all $\mu>0$. 


\section{MAIN RESULTS}

Let $K$ be a nonempty closed geodesic convex subset of $\mathbb{M}$ and $S, T: K \rightarrow K$ be mappings such that $T$ and $S$ have at least one common fixed point. We use $\operatorname{Fix}(S, T)$ to denote the set of common fixed points of the mappings $S$ and $T$. Motivated by Algorithms 1.1 and 1.2, we design $\mathrm{S}$-iteration techniques in terms of exponential mappings for solving the common fixed point problem of the nonexpansive mappings $T$ and $S$ as follows:

\section{Algorithm 3.1. (S-iteration of rank 2):}

Initialization: Select an arbitrary starting point $x_{0} \in K$,

Iterative step: Given a current iterate $x_{n}$, calculate the next iterate as follows:

$$
\left\{\begin{array}{l}
x_{n+1}=\exp _{S x_{n}}\left(1-\alpha_{n}\right) \exp _{S x_{n}}^{-1} T\left(y_{n}\right), \\
y_{n}=\exp _{x_{n}}\left(1-\beta_{n}\right) \exp _{x_{n}}^{-1} T\left(x_{n}\right), \quad \forall n \in \mathbb{N}_{0},
\end{array}\right.
$$

where $\left\{\alpha_{n}\right\}$ and $\left\{\beta_{n}\right\}$ are sequences of scalars in $(0,1)$.

\section{Algorithm 3.2. (S-iteration of rank 3):}

Initialization: Select an arbitrary starting point $x_{0} \in K$,

Iterative step: Given the current iterate $x_{n}$, calculate the next iterate as follows:

$$
\left\{\begin{array}{l}
x_{n+1}=\exp _{S x_{n}}\left(1-\alpha_{n}\right) \exp _{S x_{n}}^{-1} T\left(y_{n}\right), \\
y_{n}=\exp _{S x_{n}}\left(1-\beta_{n}\right) \exp _{S x_{n}}^{-1} T\left(z_{n}\right), \\
z_{n}=\exp _{x_{n}}\left(1-\delta_{n}\right) \exp _{x_{n}}^{-1} T\left(x_{n}\right), \quad \forall n \in \mathbb{N}_{0},
\end{array}\right.
$$

where $\left\{\alpha_{n}\right\},\left\{\beta_{n}\right\}$ and $\left\{\delta_{n}\right\}$ are the sequences of scalars in $(0,1)$.

If $S=T$, then Algorithm 3.1 reduces to

$$
\left\{\begin{array}{l}
x_{n+1}=\exp _{T x_{n}}\left(1-\alpha_{n}\right) \exp _{T x_{n}}^{-1} T\left(y_{n}\right), \\
y_{n}=\exp _{x_{n}}\left(1-\beta_{n}\right) \exp _{x_{n}}^{-1} T\left(x_{n}\right), \quad \forall n \in \mathbb{N}_{0},
\end{array}\right.
$$

whereas Algorithm 3.2 reduces to

$$
\left\{\begin{array}{l}
x_{n+1}=\exp _{T x_{n}}\left(1-\alpha_{n}\right) \exp _{T x_{n}}^{-1} T\left(y_{n}\right), \\
y_{n}=\exp _{T x_{n}}\left(1-\beta_{n}\right) \exp _{T x_{n}}^{-1} T\left(z_{n}\right), \\
z_{n}=\exp _{x_{n}}\left(1-\delta_{n}\right) \exp _{x_{n}}^{-1} T\left(x_{n}\right), \quad \forall n \in \mathbb{N}_{0} .
\end{array}\right.
$$

It can be easily seen that Algorithms 3.1 and 3.2 are different. One iterative structure can not be obtained from other. Thus, the S-iteration process of rank 2 and rank 3 are two independent S-iterative algorithms.

In order to establish convergence theorem for Algorithm 3.2, we recall following definition and lemma.

Definition 3.1. Let $X$ be a complete metric space and $F$ be a nonempty subset of $X$. A sequence $\left\{x_{n}\right\} \subset X$ is called Féjer convergent to $F$ if

$$
d\left(x_{n+1}, y\right) \leq d\left(x_{n}, y\right) \text { for any } y \in F \text { and any } n \in \mathbb{N}_{0} .
$$

Lemma 3.1. Let $X$ be a complete metric space. If $\left\{x_{n}\right\} \subset X$ is Féjer convergent to a nonempty set $F \subset X$, then $\left\{x_{n}\right\}$ is bounded. Moreover, if a cluster point $x$ of $\left\{x_{n}\right\}$ belongs to $F$, then $\left\{x_{n}\right\}$ converges to $x$. 
We now ready to establish the convergence theorem for Algorithm 3.2.

Theorem 3.1. Let $K$ be a closed geodesic convex subset of a Hadamard manifold $\mathbb{M}$ and $T$ : $K \rightarrow K$ a nonexpansive mapping with $\mathbb{F}=F i x(S, T) \neq \emptyset$. Suppose that $\left\{\alpha_{n}\right\},\left\{\beta_{n}\right\}$ and $\left\{\delta_{n}\right\}$ are sequences of scalars such that $0<\underline{a} \leq \alpha_{n} \leq \bar{a}<1,0<\underline{b} \leq \beta_{n} \leq \bar{b}<1$ and $0<\underline{c} \leq \delta_{n} \leq \bar{c}<1$ for all $n \in \mathbb{N}_{0}$. Let $x_{0} \in K$ and $\left\{x_{n}\right\}$ be the sequence generated by Algorithm 3.2. Then $\left\{x_{n}\right\}$ converges to a common fixed point of $S$ and $T$.

Proof. Since $K$ is a closed geodesic convex subset of $\mathbb{M}$, we find that $K$ itself is a complete metric space. Using Lemma 3.1, it is sufficient to prove that $\left\{x_{n}\right\}$ is Fejér convergent to $\mathbb{F}$ and that all cluster point of $\left\{x_{n}\right\}$ belongs to $\mathbb{F}$. Now, for any $n \in \mathbb{N}_{0}$, let $p \in \mathbb{F}$ be fixed and $\gamma_{1}$ be the geodesic joining $S x_{n}$ to $T y_{n}, \gamma_{2}$ be the geodesic joining $S x_{n}$ to $T z_{n}$ and $\gamma_{3}$ be the geodesic joining $x_{n}$ to $T x_{n}$. Then $x_{n+1}=\gamma_{1}\left(1-\alpha_{n}\right), y_{n}=\gamma_{2}\left(1-\beta_{n}\right)$ and $z_{n}=\gamma_{3}\left(1-\delta_{n}\right)$.

Using the geodesic convexity of distance function and the nonexpansivity of $T$ and $S$, we have

$$
\begin{aligned}
d\left(x_{n+1}, p\right) & =d\left(\gamma_{1}\left(1-\alpha_{n}\right), p\right) \\
& \leq \alpha_{n} d\left(S x_{n}, p\right)+\left(1-\alpha_{n}\right) d\left(T y_{n}, p\right) \\
& \leq \alpha_{n} d\left(x_{n}, p\right)+\left(1-\alpha_{n}\right) d\left(y_{n}, p\right) \\
d\left(y_{n}, p\right) & =d\left(\gamma_{2}\left(1-\beta_{n}\right), p\right) \\
& \leq \beta_{n} d\left(S x_{n}, p\right)+\left(1-\beta_{n}\right) d\left(T z_{n}, p\right) \\
& \leq \beta_{n} d\left(x_{n}, p\right)+\left(1-\beta_{n}\right) d\left(z_{n}, p\right)
\end{aligned}
$$

and

$$
\begin{aligned}
d\left(z_{n}, p\right) & =d\left(\gamma_{3}\left(1-\delta_{n}\right), p\right) \\
& \leq \delta_{n} d\left(x_{n}, p\right)+\left(1-\delta_{n}\right) d\left(T x_{n}, p\right) \\
& \leq \delta_{n} d\left(x_{n}, p\right)+\left(1-\delta_{n}\right) d\left(x_{n}, p\right) \\
& =d\left(x_{n}, p\right) .
\end{aligned}
$$

Combining inequalities (3.6) and (3.7), we obtain

$$
d\left(y_{n}, p\right) \leq \beta_{n} d\left(x_{n}, p\right)+\left(1-\beta_{n}\right) d\left(x_{n}, p\right)=d\left(x_{n}, p\right) .
$$

This together with inequality (3.5) yields

$$
d\left(x_{n+1}, p\right) \leq \alpha_{n} d\left(x_{n}, p\right)+\left(1-\alpha_{n}\right) d\left(x_{n}, p\right)=d\left(x_{n}, p\right) .
$$

Hence, $\left\{x_{n}\right\}$ is Fejér convergent to $\mathbb{F}$. From Lemma 2.1, we have that $\left\{x_{n}\right\}$ is bounded. Let $\bar{x}$ be a cluster point of $\left\{x_{n}\right\}$. Then there exists a subsequence $\left\{n_{k}\right\}$ of $n$ such that $x_{n_{k}} \rightarrow \bar{x}$. Now, we show that

$$
\lim _{n \rightarrow \infty} d\left(x_{n}, T x_{n}\right)=0 .
$$

For arbitrary $p \in \mathbb{F}$, set $n \in \mathbb{N}_{0}$. Let $\Delta(l, q, p)$ be a geodesic triangle with vertices $l=S x_{n}$, $q=T y_{n}$ and $p$. From Lemma 2.1, there exists a comparison triangle $\Delta\left(l^{\prime}, q^{\prime}, p^{\prime}\right)$ which conserves 
the length of edges. Also, we have $x_{n+1}=\gamma_{1}\left(1-\alpha_{n}\right)$. Set $x_{n+1}^{\prime}=\alpha_{n} l^{\prime}+\left(1-\alpha_{n}\right) q^{\prime}$ as its comparison point. By Lemma 2.2, we have

$$
\begin{aligned}
d^{2}\left(x_{n+1}, p\right) & \leq\left\|x_{n+1}^{\prime}-p^{\prime}\right\|^{2} \\
& =\left\|\alpha_{n}\left(l^{\prime}-p^{\prime}\right)+\left(1-\alpha_{n}\right)\left(q^{\prime}-p^{\prime}\right)\right\|^{2} \\
& =\alpha_{n}\left\|l^{\prime}-p^{\prime}\right\|^{2}+\left(1-\alpha_{n}\right)\left\|p^{\prime}-q^{\prime}\right\|^{2}-\alpha_{n}\left(1-\alpha_{n}\right)\left\|l^{\prime}-q^{\prime}\right\|^{2} \\
& =\alpha_{n} d^{2}\left(S x_{n}, p\right)+\left(1-\alpha_{n}\right) d^{2}\left(T y_{n}, p\right)-\alpha_{n}\left(1-\alpha_{n}\right) d^{2}\left(S x_{n}, T y_{n}\right) \\
& \leq \alpha_{n} d^{2}\left(x_{n}, p\right)+\left(1-\alpha_{n}\right) d^{2}\left(y_{n}, p\right)-\alpha_{n}\left(1-\alpha_{n}\right) d^{2}\left(S x_{n}, T y_{n}\right) \\
& \leq d^{2}\left(x_{n}, p\right)-\alpha_{n}\left(1-\alpha_{n}\right) d^{2}\left(S x_{n}, T y_{n}\right), \quad \forall n \in \mathbb{N}_{0},
\end{aligned}
$$

which gives that

$$
\alpha_{n}\left(1-\alpha_{n}\right) d^{2}\left(S x_{n}, T y_{n}\right) \leq d^{2}\left(x_{n}, p\right)-d^{2}\left(x_{n+1}, p\right), \quad \forall n \in \mathbb{N}_{0} .
$$

Observe that $\underline{a}(1-\bar{a}) \leq \alpha_{n}\left(1-\alpha_{n}\right)$ for all $n \in \mathbb{N}_{0}$. Summing up the above inequality from $i=0$ to $i=n$, we have

$$
\underline{a}(1-\bar{a}) \sum_{i=0}^{n} d^{2}\left(S x_{i}, T y_{i}\right) \leq d^{2}\left(x_{0}, p\right)-d^{2}\left(x_{n+1}, p\right), \quad \forall n \in \mathbb{N}_{0} .
$$

Letting $n \rightarrow \infty$, we obtain

$$
\underline{a}(1-\bar{a}) \sum_{n=0}^{\infty} d^{2}\left(S x_{n}, T y_{n}\right) \leq d^{2}\left(x_{0}, p\right)<\infty .
$$

This implies that

$$
\lim _{n \rightarrow \infty} d\left(S x_{n}, T y_{n}\right)=0 .
$$

Now, let $\Delta(l, s, p)$ be a geodesic triangle with vertices $l=S x_{n}, s=T z_{n}$ and $p$. From Lemma 2.1, there exists a comparison triangle $\Delta\left(l^{\prime}, s^{\prime}, p^{\prime}\right)$ which conserves the length of edges. Also, we have $y_{n}=\gamma_{2}\left(1-\beta_{n}\right)$. Set $y_{n}^{\prime}=\beta_{n} l^{\prime}+\left(1-\beta_{n}\right) s^{\prime}$ as its comparison point. By Lemma 2.2, we have

$$
\begin{aligned}
d^{2}\left(y_{n}, p\right) & \leq\left\|y_{n}^{\prime}-p^{\prime}\right\|^{2} \\
& =\left\|\beta_{n}\left(l^{\prime}-p^{\prime}\right)+\left(1-\beta_{n}\right)\left(s^{\prime}-p^{\prime}\right)\right\|^{2} \\
& =\beta_{n}\left\|l^{\prime}-p^{\prime}\right\|^{2}+\left(1-\beta_{n}\right)\left\|p^{\prime}-s^{\prime}\right\|^{2}-\beta_{n}\left(1-\beta_{n}\right)\left\|l^{\prime}-s^{\prime}\right\|^{2} \\
& =\beta_{n} d^{2}\left(S x_{n}, p\right)+\left(1-\beta_{n}\right) d^{2}\left(T z_{n}, p\right)-\beta_{n}\left(1-\beta_{n}\right) d^{2}\left(S x_{n}, T z_{n}\right) \\
& \leq \beta_{n} d^{2}\left(x_{n}, p\right)+\left(1-\beta_{n}\right) d^{2}\left(z_{n}, p\right)-\beta_{n}\left(1-\beta_{n}\right) d^{2}\left(S x_{n}, T z_{n}\right) \\
& \leq d^{2}\left(x_{n}, p\right)-\beta_{n}\left(1-\beta_{n}\right) d^{2}\left(S x_{n}, T z_{n}\right), \quad \forall n \in \mathbb{N}_{0} .
\end{aligned}
$$

From (3.9), we have

$$
\begin{aligned}
d^{2}\left(x_{n+1}, p\right) \leq & \alpha_{n} d^{2}\left(x_{n}, p\right)+\left(1-\alpha_{n}\right) d^{2}\left(x_{n}, p\right)-\left(1-\alpha_{n}\right) \beta_{n}\left(1-\beta_{n}\right) d^{2}\left(S x_{n}, T z_{n}\right) \\
& -\alpha_{n}\left(1-\alpha_{n}\right) d^{2}\left(S x_{n}, T y_{n}\right) \\
= & d^{2}\left(x_{n}, p\right)-\left(1-\alpha_{n}\right) \beta_{n}\left(1-\beta_{n}\right) d^{2}\left(S x_{n}, T z_{n}\right)-\alpha_{n}\left(1-\alpha_{n}\right) d^{2}\left(S x_{n}, T y_{n}\right) .
\end{aligned}
$$


Observe that $\underline{b}(1-\bar{a})(1-\bar{b}) \leq\left(1-\alpha_{n}\right) \beta_{n}\left(1-\beta_{n}\right)$ for all $n \in \mathbb{N}_{0}$. Hence, summing up the above inequality from $i=0$ to $i=n$, we get

$$
\underline{b}(1-\bar{a})(1-\bar{b}) \sum_{i=0}^{n} d^{2}\left(S x_{i}, T z_{i}\right) \leq d^{2}\left(x_{0}, p\right)-d^{2}\left(x_{n+1}, p\right)-\sum_{i=0}^{n} d^{2}\left(S x_{i}, T y_{i}\right) .
$$

Letting $n \rightarrow \infty$, we obtain

$$
\underline{b}(1-\bar{a})(1-\bar{b}) \sum_{n=0}^{\infty} d^{2}\left(S x_{n}, T z_{n}\right) \leq d^{2}\left(x_{0}, p\right)<\infty .
$$

It follows that

$$
\lim _{n \rightarrow \infty} d\left(S x_{n}, T z_{n}\right)=0 .
$$

Moreover, let $\Delta\left(x_{n}, e, p\right)$ be a geodesic triangle with vertices $x_{n}, e=T x_{n}$ and $p$. From lemma 2.1, there exists a comparison triangle $\Delta\left(x_{n}^{\prime}, e^{\prime}, p^{\prime}\right)$ which conserves the length of edges. Also, we have $z_{n}=\gamma_{3}\left(1-\delta_{n}\right)$ and set $z_{n}^{\prime}=\delta_{n} x_{n}^{\prime}+\left(1-\delta_{n}\right) e^{\prime}$. Similarly, by Lemma 2.2, we have

$$
\begin{aligned}
d^{2}\left(z_{n}, p\right) & \leq\left\|z_{n}^{\prime}-p\right\|^{2} \\
& =\left\|\delta_{n}\left(x_{n}^{\prime}-p^{\prime}\right)+\left(1-\delta_{n}\right)\left(e^{\prime}-p^{\prime}\right)\right\|^{2} \\
& =\delta_{n}\left\|x_{n}^{\prime}-p\right\|^{2}+\left(1-\delta_{n}\right)\left\|e^{\prime}-p^{\prime}\right\|^{2}-\delta_{n}\left(1-\delta_{n}\right)\left\|x_{n}^{\prime}-e^{\prime}\right\|^{2} \\
& =\delta_{n} d^{2}\left(x_{n}, p\right)+\left(1-\delta_{n}\right) d^{2}\left(T x_{n}, p\right)-\delta_{n}\left(1-\delta_{n}\right) d^{2}\left(x_{n}, T x_{n}\right) \\
& \leq \delta_{n} d^{2}\left(x_{n}, p\right)+\left(1-\delta_{n}\right) d^{2}\left(x_{n}, p\right)-\delta_{n}\left(1-\delta_{n}\right) d^{2}\left(x_{n}, T x_{n}\right) \\
& \leq d^{2}\left(x_{n}, p\right)-\delta_{n}\left(1-\delta_{n}\right) d^{2}\left(x_{n}, T x_{n}\right) .
\end{aligned}
$$

Combining (3.10) and (3.11), we obtain

$$
\begin{aligned}
& d^{2}\left(y_{n}, p\right) \\
& \leq \beta_{n} d^{2}\left(x_{n}, p\right)+\left(1-\beta_{n}\right)\left[d^{2}\left(x_{n}, p\right)-\delta_{n}\left(1-\delta_{n}\right) d^{2}\left(x_{n}, T x_{n}\right)\right]-\beta_{n}\left(1-\beta_{n}\right) d^{2}\left(S x_{n}, T z_{n}\right) \\
& \leq \beta_{n} d^{2}\left(x_{n}, p\right)+\left(1-\beta_{n}\right) d^{2}\left(x_{n}, p\right)-\delta_{n}\left(1-\beta_{n}\right)\left(1-\delta_{n}\right) d^{2}\left(x_{n}, T x_{n}\right) \\
& \quad-\beta_{n}\left(1-\beta_{n}\right) d^{2}\left(S x_{n}, T z_{n}\right) \\
& \leq d^{2}\left(x_{n}, p\right)-\delta_{n}\left(1-\beta_{n}\right)\left(1-\delta_{n}\right) d^{2}\left(x_{n}, T x_{n}\right)-\beta_{n}\left(1-\beta_{n}\right) d^{2}\left(S x_{n}, T z_{n}\right) .
\end{aligned}
$$

By combining (3.9) and (3.12), we obtain that

$$
\begin{aligned}
& d^{2}\left(x_{n+1}, p\right) \\
& \leq \alpha_{n} d^{2}\left(x_{n}, p\right)+\left(1-\alpha_{n}\right)\left[d^{2}\left(x_{n}, p\right)-\delta_{n}\left(1-\beta_{n}\right)\left(1-\delta_{n}\right) d^{2}\left(x_{n}, T x_{n}\right)\right. \\
& \left.-\beta_{n}\left(1-\beta_{n}\right) d^{2}\left(S x_{n}, T z_{n}\right)\right]-\alpha_{n}\left(1-\alpha_{n}\right) d^{2}\left(S x_{n}, T y_{n}\right) \\
& \leq \alpha_{n} d^{2}\left(x_{n}, p\right)+\left(1-\alpha_{n}\right) d^{2}\left(x_{n}, p\right)-\delta_{n}\left(1-\alpha_{n}\right)\left(1-\beta_{n}\right)\left(1-\delta_{n}\right) d^{2}\left(x_{n}, T x_{n}\right) \\
& -\beta_{n}\left(1-\alpha_{n}\right)\left(1-\beta_{n}\right) d^{2}\left(S x_{n}, T z_{n}\right)-\alpha_{n}\left(1-\alpha_{n}\right) d^{2}\left(S x_{n}, T y_{n}\right) \\
& \leq d^{2}\left(x_{n}, p\right)-\delta_{n}\left(1-\alpha_{n}\right)\left(1-\beta_{n}\right)\left(1-\delta_{n}\right) d^{2}\left(x_{n}, T x_{n}\right)-\beta_{n}\left(1-\alpha_{n}\right)\left(1-\beta_{n}\right) d^{2}\left(S x_{n}, T z_{n}\right) \\
& -\alpha_{n}\left(1-\alpha_{n}\right) d^{2}\left(S x_{n}, T y_{n}\right) \quad \forall n \in \mathbb{N}_{0} .
\end{aligned}
$$

Notice that

$$
\underline{c}(1-\bar{a})(1-\bar{b})(1-\bar{c}) \leq \delta_{n}\left(1-\alpha_{n}\right)\left(1-\beta_{n}\right)\left(1-\delta_{n}\right)
$$


for all $n \in \mathbb{N}_{0}$. Taking the sum of the above inequality from $i=0$ to $i=n$, we observe that

$$
\begin{aligned}
& \underline{c}(1-\bar{a})(1-\bar{b})(1-\bar{c}) \sum_{i=0}^{n} d^{2}\left(x_{i}, T x_{i}\right) \\
& \leq d^{2}\left(x_{0}, p\right)-d^{2}\left(x_{n+1}, p\right)-\sum_{i=0}^{n} \beta_{n}\left(1-\alpha_{n}\right)\left(1-\beta_{n}\right) d^{2}\left(S x_{i}, T z_{i}\right)-\sum_{i=0}^{n} \alpha_{n}\left(1-\alpha_{n}\right) d^{2}\left(S x_{i}, T y_{i}\right) .
\end{aligned}
$$

Letting $n \rightarrow \infty$, we obtain

$$
\underline{c}(1-\bar{a})(1-\bar{b})(1-\bar{c}) \sum_{n=0}^{\infty} d^{2}\left(x_{n}, T x_{n}\right) \leq d^{2}\left(x_{0}, p\right)<\infty .
$$

It follows that

$$
\lim _{n \rightarrow \infty} d\left(x_{n}, T x_{n}\right)=0
$$

By the geodesic convexity of Riemannian distance, we have

$$
\begin{aligned}
d\left(z_{n}, x_{n}\right) & \leq \delta_{n} d\left(x_{n}, x_{n}\right)+\left(1-\delta_{n}\right) d\left(T x_{n}, x_{n}\right) \\
& =\left(1-\delta_{n}\right) d\left(T x_{n}, x_{n}\right) \\
& \leq d\left(T x_{n}, x_{n}\right) \rightarrow 0 \text { as } n \rightarrow \infty
\end{aligned}
$$

Since $T$ is nonexpansive, we have

$$
\begin{aligned}
d\left(z_{n}, T z_{n}\right) & \leq d\left(z_{n}, x_{n}\right)+d\left(x_{n}, T x_{n}\right)+d\left(T x_{n}, T z_{n}\right) \\
& \leq d\left(x_{n}, z_{n}\right)+d\left(x_{n}, T x_{n}\right)+d\left(x_{n}, z_{n}\right) \\
& \leq 2 d\left(x_{n}, z_{n}\right)+d\left(x_{n}, T x_{n}\right) \rightarrow 0 \text { as } n \rightarrow \infty .
\end{aligned}
$$

On the other hand,

$$
d\left(x_{n}, S x_{n}\right) \leq d\left(S x_{n}, T z_{n}\right)+d\left(T z_{n}, z_{n}\right)+d\left(z_{n}, x_{n}\right) .
$$

By taking limit as $n \rightarrow \infty$. Then, by combining above three inequalities, it follows that

$$
\lim _{n \rightarrow \infty} d\left(x_{n}, S x_{n}\right) \leq \lim _{n \rightarrow \infty} d\left(S x_{n}, T z_{n}\right)+\lim _{n \rightarrow \infty} d\left(T z_{n}, z_{n}\right)+\lim _{n \rightarrow \infty} d\left(z_{n}, x_{n}\right)=0 .
$$

Thus, we deduce that

$$
\begin{aligned}
d(\bar{x}, T \bar{x}) & \leq d\left(\bar{x}, x_{n_{k}}\right)+d\left(x_{n_{k}}, T x_{n_{k}}\right)+d\left(T x_{n_{k}}, T \bar{x}\right) \\
& \leq 2 d\left(x_{n_{k}}, \bar{x}\right)+d\left(x_{n_{k}}, T x_{n_{k}}\right),
\end{aligned}
$$

and

$$
\begin{aligned}
d(\bar{x}, S \bar{x}) & \leq d\left(\bar{x}, x_{n_{k}}\right)+d\left(x_{n_{k}}, S x_{n_{k}}\right)+d\left(S x_{n_{k}}, S \bar{x}\right) \\
& \leq 2 d\left(x_{n_{k}}, \bar{x}\right)+d\left(x_{n_{k}}, S x_{n_{k}}\right) .
\end{aligned}
$$

Taking limit as $k \rightarrow \infty$, we evaluate that $d(\bar{x}, T \bar{x})=0$ and $d(\bar{x}, S \bar{x})=0$, which means that $\bar{x} \in$ $\operatorname{Fix}(T) \cap \operatorname{Fix}(S)$. The proof is complete.

Remark 3.1. Theorem 3.1 is an extension of Corollary 1 of [17] from a Hilbert space to the manifold for finding common fixed points of nonexpansive mappings.

Now, we establish a convergence theorem of Algorithm 3.3 with some different assumptions on scalars $\left\{\alpha_{n}\right\}$ and $\left\{\beta_{n}\right\}$. 
Theorem 3.2. Let $K$ be a closed geodesic convex subset of a Hadamard manifold $\mathbb{M}$ and $T$ : $K \rightarrow K$ a nonexpansive mapping with $\mathrm{Fix}(T) \neq \emptyset$. Suppose that $\left\{\alpha_{n}\right\}$ and $\left\{\beta_{n}\right\}$ are sequences of scalars in $(0,1)$ such that

$$
\sum_{n=0}^{\infty} \beta_{n}\left(1-\alpha_{n}\right)\left(1-\beta_{n}\right)=+\infty
$$

Let $x_{0} \in K$ and $\left\{x_{n}\right\}$ be the sequence generated by Algorithm 3.3. Then $\left\{x_{n}\right\}$ converges to a fixed point of $T$.

Proof. Since $K$ is a closed geodesic convex subset of $\mathbb{M}$, then $K$ itself is a complete metric space. By replacing $T=S$ in Theorem 3.1, we easily get that $\left\{x_{n}\right\}$ is Fejér convergent to Fix $(T)$. Hence, Lemma 3.1 insures that $\left\{x_{n}\right\}$ is bounded. We just need to show that an arbitrary cluster point of $\left\{x_{n}\right\}$ belongs to $\operatorname{Fix}(T)$.

Now, we show that

$$
\lim _{n \rightarrow \infty} d\left(x_{n}, T x_{n}\right)=0 .
$$

For this, let $p \in F$ and $n \in \mathbb{N}_{0}$. Let $\Delta(l, q, p)$ be the geodesic triangle with vertices $l=T x_{n}$, $q=T y_{n}$ and $p$. From Lemma 2.1, there exists a comparison triangle $\Delta\left(l^{\prime}, q^{\prime}, p^{\prime}\right)$ which conserves the length of edges. Also we have $x_{n+1}=\gamma_{1}\left(1-\alpha_{n}\right)$. Set $x_{n+1}^{\prime}=\alpha_{n} l^{\prime}+\left(1-\alpha_{n}\right) q^{\prime}$ as its comparison point. By Lemma 2.2, we have

$$
\begin{aligned}
d^{2}\left(x_{n+1}, p\right) & \leq\left\|x_{n+1}^{\prime}-p^{\prime}\right\|^{2} \\
& =\left\|\alpha_{n}\left(l^{\prime}-p^{\prime}\right)+\left(1-\alpha_{n}\right)\left(q^{\prime}-p^{\prime}\right)\right\|^{2} \\
& =\alpha_{n}\left\|l^{\prime}-p^{\prime}\right\|^{2}+\left(1-\alpha_{n}\right)\left\|p^{\prime}-q^{\prime}\right\|^{2}-\alpha_{n}\left(1-\alpha_{n}\right)\left\|l^{\prime}-q^{\prime}\right\|^{2} \\
& =\alpha_{n} d^{2}\left(T x_{n}, p\right)+\left(1-\alpha_{n}\right) d^{2}\left(T y_{n}, p\right)-\alpha_{n}\left(1-\alpha_{n}\right) d^{2}\left(T x_{n}, T y_{n}\right) \\
& \leq \alpha_{n} d^{2}\left(x_{n}, p\right)+\left(1-\alpha_{n}\right) d^{2}\left(y_{n}, p\right)-\alpha_{n}\left(1-\alpha_{n}\right) d^{2}\left(T x_{n}, T y_{n}\right) \\
& \leq \alpha_{n} d^{2}\left(x_{n}, p\right)+\left(1-\alpha_{n}\right) d^{2}\left(y_{n}, p\right), \quad \forall n \in \mathbb{N}_{0} .
\end{aligned}
$$

Now, let $\Delta(l, s, p)$ be the geodesic triangle with vertices $l=T x_{n}, s=x_{n}$ and $p$. From Lemma 2.1, there exists a comparison triangle $\Delta\left(l^{\prime}, s^{\prime}, p^{\prime}\right)$ which conserves the length of edges. Also we have $y_{n}=\gamma_{2}\left(1-\beta_{n}\right)$. Set $y_{n}^{\prime}=\beta_{n} l^{\prime}+\left(1-\beta_{n}\right) s^{\prime}$ as its comparison point. By Lemma 2.2, we have

$$
\begin{aligned}
d^{2}\left(y_{n}, p\right) & \leq\left\|y_{n}^{\prime}-p^{\prime}\right\|^{2} \\
& =\left\|\beta_{n}\left(l^{\prime}-p^{\prime}\right)+\left(1-\beta_{n}\right)\left(q^{\prime}-p^{\prime}\right)\right\|^{2} \\
& =\beta_{n}\left\|l^{\prime}-p^{\prime}\right\|^{2}+\left(1-\beta_{n}\right)\left\|p^{\prime}-q^{\prime}\right\|^{2}-\beta_{n}\left(1-\beta_{n}\right)\left\|l^{\prime}-q^{\prime}\right\|^{2} \\
& =\beta_{n} d^{2}\left(T x_{n}, p\right)+\left(1-\beta_{n}\right) d^{2}\left(x_{n}, p\right)-\beta_{n}\left(1-\beta_{n}\right) d^{2}\left(T x_{n}, x_{n}\right) \\
& \leq \beta_{n} d^{2}\left(x_{n}, p\right)+\left(1-\beta_{n}\right) d^{2}\left(x_{n}, p\right)-\beta_{n}\left(1-\beta_{n}\right) d^{2}\left(T x_{n}, x_{n}\right) \\
& =d^{2}\left(x_{n}, p\right)-\beta_{n}\left(1-\beta_{n}\right) d^{2}\left(T x_{n}, x_{n}\right), \quad \forall n \in \mathbb{N}_{0} .
\end{aligned}
$$

This together with (3.14) implies

$$
d^{2}\left(x_{n+1}, p\right) \leq \alpha_{n} d^{2}\left(x_{n}, p\right)+\left(1-\alpha_{n}\right)\left(d^{2}\left(x_{n}, p\right)-\beta_{n}\left(1-\beta_{n}\right) d^{2}\left(T x_{n}, x_{n}\right)\right) .
$$


Observe that

$$
\beta_{n}\left(1-\alpha_{n}\right)\left(1-\beta_{n}\right) d^{2}\left(T x_{n}, x_{n}\right) \leq d^{2}\left(x_{n}, p\right)-d^{2}\left(x_{n+1}, p\right), \quad \forall n \in \mathbb{N}_{0} .
$$

Summing the above inequality from $i=0$ to $n$, we get

$$
\sum_{i=0}^{n} \beta_{i}\left(1-\alpha_{i}\right)\left(1-\beta_{i}\right) d^{2}\left(T x_{i}, x_{i}\right) \leq d^{2}\left(x_{0}, p\right)
$$

which gives us that

$$
\sum_{i=0}^{\infty} \beta_{i}\left(1-\alpha_{i}\right)\left(1-\beta_{i}\right) d^{2}\left(T x_{i}, x_{i}\right) \leq d^{2}\left(x_{0}, p\right)<+\infty .
$$

We claim that $\liminf _{n \rightarrow \infty} d\left(T x_{n}, x_{n}\right)=0$. Let, on the contrary, $d\left(T x_{n}, x_{n}\right) \geq \bar{a}$ for all $n \in \mathbb{N}_{0}$ for some $\bar{a}>0$. Then

$$
\sum_{i=0}^{\infty} \beta_{i}\left(1-\alpha_{i}\right)\left(1-\beta_{i}\right) d^{2}\left(T x_{n}, x_{n}\right) \geq \bar{a} \sum_{i=0}^{\infty} \beta_{i}\left(1-\alpha_{i}\right)\left(1-\beta_{i}\right)=+\infty,
$$

which contradict (3.17). Thus,

$$
\liminf _{n \rightarrow \infty} d\left(T x_{n}, x_{n}\right)=0
$$

On the other hand, by using the nonexpansivity of $T$ and the convexity of the distance function, we get

$$
\begin{aligned}
d\left(x_{n+1}, T x_{n+1}\right) & \leq \alpha_{n} d\left(T x_{n}, T x_{n+1}\right)+\left(1-\alpha_{n}\right) d\left(T y_{n}, T x_{n+1}\right) \\
& \leq \alpha_{n} d\left(x_{n}, x_{n+1}\right)+\left(1-\alpha_{n}\right) d\left(y_{n}, x_{n+1}\right) .
\end{aligned}
$$

Note

$$
\begin{aligned}
d\left(x_{n}, x_{n+1}\right) & \leq \alpha_{n} d\left(T x_{n}, x_{n}\right)+\left(1-\alpha_{n}\right) d\left(T y_{n}, x_{n}\right) \\
& \leq \alpha_{n} d\left(T x_{n}, x_{n}\right)+\left(1-\alpha_{n}\right)\left(d\left(y_{n}, x_{n}\right)+d\left(T x_{n}, x_{n}\right)\right) \\
& \leq \alpha_{n} d\left(T x_{n}, x_{n}\right)+\left(1-\alpha_{n}\right)\left(\left(1-\beta_{n}\right) d\left(T x_{n}, x_{n}\right)+d\left(T x_{n}, x_{n}\right)\right) \\
& \leq\left(1+\left(1-\alpha_{n}\right)\left(1-\beta_{n}\right)\right) d\left(T x_{n}, x_{n}\right)
\end{aligned}
$$

and

$$
\begin{aligned}
d\left(y_{n}, x_{n+1}\right) \leq & \alpha_{n} d\left(T x_{n}, y_{n}\right)+\left(1-\alpha_{n}\right) d\left(T y_{n}, y_{n}\right) \\
\leq & \alpha_{n}\left(\beta_{n} d\left(T x_{n}, x_{n}\right)+\left(1-\beta_{n}\right) d\left(T x_{n}, T x_{n}\right)\right)+\left(1-\alpha_{n}\right)\left(\beta_{n} d\left(x_{n}, T y_{n}\right)\right. \\
& \left.+\left(1-\beta_{n}\right) d\left(T x_{n}, T y_{n}\right)\right) \\
\leq & \alpha_{n} \beta_{n} d\left(T x_{n}, x_{n}\right)+\left(1-\alpha_{n}\right)\left(\beta_{n} d\left(T x_{n}, T y_{n}\right)+\beta_{n} d\left(T x_{n}, x_{n}\right)+\left(1-\beta_{n}\right) d\left(x_{n}, y_{n}\right)\right) \\
= & \alpha_{n} \beta_{n} d\left(T x_{n}, x_{n}\right)+\left(1-\alpha_{n}\right)\left(\beta_{n} d\left(T x_{n}, x_{n}\right)+d\left(x_{n}, y_{n}\right)\right) \\
= & \left(1+\alpha_{n} \beta_{n}-\alpha_{n}\right) d\left(T x_{n}, x_{n}\right) .
\end{aligned}
$$

By combining inequalities (3.19), (3.20) and (3.21), we obtain

$$
\begin{aligned}
& d\left(x_{n+1}, T x_{n+1}\right) \\
& \left.\leq \alpha_{n}\left(\left(1+\left(1-\alpha_{n}\right)\left(1-\beta_{n}\right)\right) d\left(T x_{n}, x_{n}\right)\right)+\left(1-\alpha_{n}\right)\left(\left(1+\alpha_{n} \beta_{n}-\alpha_{n}\right) d\left(T x_{n}, x_{n}\right)\right)\right) \\
& =d\left(T x_{n}, x_{n}\right) .
\end{aligned}
$$


This implies that $\left\{d\left(x_{n}, T x_{n}\right)\right\}$ is a monotonic decreasing sequence. This together with equality (3.18) insures that

$$
\lim _{n \rightarrow \infty} d\left(T x_{n}, x_{n}\right)=0 .
$$

Since $\left\{x_{n}\right\}$ is bounded, then there exists a subsequence $\left\{x_{n_{k}}\right\}$ of $\left\{x_{n}\right\}$ such that $x_{n_{k}} \rightarrow \bar{x}$ as $k \rightarrow \infty$, where $\bar{x}$ be the cluster point of the sequence $\left\{x_{n}\right\}$. Thus, we can conclude that

$$
\begin{aligned}
d(\bar{x}, T \bar{x}) & \leq d\left(\bar{x}, x_{n_{k}}\right)+d\left(x_{n_{k}}, T x_{n_{k}}\right)+d\left(T x_{n_{k}}, T \bar{x}\right) \\
& \leq 2 d\left(x_{n_{k}}, \bar{x}\right)+d\left(x_{n_{k}}, T x_{n_{k}}\right) .
\end{aligned}
$$

By taking limit as $k \rightarrow \infty$, we deduce that $d(\bar{x}, T \bar{x})=0$, it implies that $\bar{x} \in F i x(T)$. This completes the proof.

Remark 3.2. Theorem 3.2 is an extension of Theorem 6.7.4 of [1] from an uniformly convex Banach space to a manifold for finding fixed points of nonexpansive mappings.

\section{NUMERICAL EXAMPLES}

Consider $\mathbb{E}^{n, 1}$, which denotes the vector space $\mathbb{R}^{n+1}$ endowed with the symmetric bilinear form (which is called the Lorentz metric) that associate to vectors $u=\left(u_{1}, \cdots, u_{n+1}\right)$ and $v=$ $\left(v_{1}, \cdots, v_{n+1}\right)$, the real number $\langle u, v\rangle$ defined by

$$
\langle u, v\rangle=\sum_{i=1}^{n} u_{i} v_{i}-u_{n+1} v_{n+1} .
$$

Then the Real Hyperbolic $n$-space $\mathbb{H}^{n}$ is the space defined by

$$
\mathbb{H}^{n}=\left\{u=\left(u_{1}, \cdots, u_{n+1}\right) \in \mathbb{E}^{n, 1}:\langle u, u\rangle=-1, u_{n+1}>0\right\},
$$

which is the upper sheet of the hyperboloid $\left\{x \in \mathbb{E}^{n, 1}:\langle x, x\rangle=-1\right\}$. Note that $x_{n+1}>0$ for any $x \in \mathbb{H}^{n}$, with equality if and only if $x_{i}=0$ for all $i=1, \cdots, n$. The metric of $\mathbb{H}^{n}$ is induced from the lorentz metric $\langle.,$.$\rangle , i,e., for u, v \in \mathbb{M}$,

$$
d(u, v)=\cosh ^{-1}(-\langle u, v\rangle)
$$

Then $\mathbb{H}^{n}$ is a Hadamard manifold with sectional curvature -1 . For more details, we refer to $[6,13]$. Normalized geodesic $\gamma: \mathbb{R} \rightarrow \mathbb{H}^{n}$ starting from $x \in \mathbb{H}^{n}$ is given by

$$
\gamma(t)=(\cosh (t)) x+(\sinh (t)) v, \quad \forall x, y \in \mathbb{H}^{n},
$$

where $v \in T_{x} \mathbb{H}^{n}$ is a unit vector, that is, $\langle v, v\rangle=1$. The exponential map can be expressed as $\exp _{x}: T_{x} \mathbb{H}^{n} \rightarrow \mathbb{H}^{n}$ such that

$$
\exp _{x}(r v)=(\cosh r) x+(\sinh r) v
$$


for any $r \in \mathbb{R}^{+}, x \in \mathbb{H}^{n}$ and any unit vector $v \in T_{x} \mathbb{H}^{n}$. To get the expression of the inverse exponential map, we write, for any $x, y \in \mathbb{H}^{n}$,

$$
\begin{aligned}
y & =\exp _{x}\left(\exp _{x}^{-1} y\right) \\
& =\exp _{x}\left(d(x, y) \frac{\exp _{x}^{-1} y}{d(x, y)}\right) \\
& =(\cosh d(x, y)) x+(\sinh d(x, y)) \frac{\exp _{x}^{-1} y}{d(x, y)} \\
& =-\langle x, y\rangle x+\frac{\left(\cosh \cosh ^{-1}(-\langle x, y\rangle)^{2}-1\right)^{\frac{1}{2}} \exp _{x}^{-1} y}{\cosh ^{-1}(-\langle x, y\rangle)} \\
& =-\langle x, y\rangle x+\frac{\left((-\langle x, y\rangle)^{2}-1\right)^{\frac{1}{2}} \exp _{x}^{-1} y}{\cosh ^{-1}(-\langle x, y\rangle)} .
\end{aligned}
$$

This implies that

$$
\exp _{x}^{-1} y=\cosh ^{-1}(-\langle x, y\rangle) \frac{y+\langle x, y\rangle x}{\sqrt{\langle x, y\rangle^{2}-1}} .
$$

Write $r(x, y)=\cosh ^{-1}(-\langle x, y\rangle)$ and $v(x, y)=\frac{y+\langle x, y\rangle x}{\sqrt{\langle x, y\rangle^{2}-1}}$. Then $\exp _{x}^{-1} y=r(x, y) \cdot v(x, y)$. Now, let

$$
\mathbb{M}=\mathbb{H}^{3}=\left\{\left(x_{1}, x_{2}, x_{3}, x_{4}\right) \in \mathbb{R}^{4}: x_{1}^{2}+x_{2}^{2}+x_{3}^{2}-x_{4}^{2}=-1, x_{4}>0\right\}
$$

and consider a nonexpansive mapping $T: \mathbb{M} \rightarrow \mathbb{M}$ given by

$$
T\left(x_{1}, x_{2}, x_{3}, x_{4}\right)=\left(-x_{1},-x_{2},-x_{3}, x_{4}\right), \quad\left(x_{1}, x_{2}, x_{3}, x_{4}\right) \in \mathbb{H}^{3} .
$$

Note that if $T\left(x_{1}, x_{2}, x_{3}, x_{4}\right)=\left(x_{1}, x_{2}, x_{3}, x_{4}\right)$, then

$$
\left(-x_{1},-x_{2},-x_{3}, x_{4}\right)=\left(x_{1}, x_{2}, x_{3}, x_{4}\right) \text {. }
$$

Therefore $(0,0,0,1) \in \mathbb{H}^{3}$ is the only fixed point of $T$. To study the convergence to a fixed point for map $T$, we take $S=T$ in Algorithm 3.1 and Algorithm 3.2. Then the following algorithms for the Mann iteration method, the S-iteration method of rank 2 and the S-iteration method of rank 3 are taken the form as follows.

Mann's algorithm [13]:

$$
\begin{gathered}
x_{n+1}=\left(\cosh \left(1-\alpha_{n}\right) r\left(x_{n}, T x_{n}\right)\right) x_{n}+\left(\sinh \left(1-\alpha_{n}\right) r\left(x_{n}, T x_{n}\right)\right) v\left(x_{n}, T x_{n}\right), \\
\left\{\begin{array}{l}
y_{n}=\left(\cosh \left(1-\alpha_{n}\right) r\left(x_{n}, T x_{n}\right)\right) x_{n}+\left(\sinh \left(1-\alpha_{n}\right) r\left(x_{n}, T x_{n}\right)\right) v\left(x_{n}, T x_{n}\right), \\
x_{n+1}=\left(\cosh \left(1-\alpha_{n}\right) r\left(T x_{n}, T y_{n}\right)\right) x_{n}+\left(\sinh \left(1-\alpha_{n}\right) r\left(T x_{n}, T y_{n}\right)\right) v\left(T x_{n}, T y_{n}\right), \forall n \in \mathbb{N}_{0},
\end{array}\right.
\end{gathered}
$$

and

$$
\left\{\begin{array}{l}
z_{n}=\left(\cosh \left(1-\alpha_{n}\right) r\left(x_{n}, T x_{n}\right)\right) x_{n}+\left(\sinh \left(1-\alpha_{n}\right) r\left(x_{n}, T x_{n}\right)\right) v\left(x_{n}, T x_{n}\right), \\
y_{n}=\left(\cosh \left(1-\alpha_{n}\right) r\left(T x_{n}, T z_{n}\right)\right) x_{n}+\left(\sinh \left(1-\alpha_{n}\right) r\left(T x_{n}, T z_{n}\right)\right) v\left(T x_{n}, T z_{n}\right), \\
x_{n+1}=\left(\cosh \left(1-\alpha_{n}\right) r\left(T x_{n}, T y_{n}\right)\right) x_{n}+\left(\sinh \left(1-\alpha_{n}\right) r\left(T x_{n}, T y_{n}\right)\right) v\left(T x_{n}, T y_{n}\right), \forall n \in \mathbb{N}_{0},
\end{array}\right.
$$

respectively. The following table denotes the error rates, where $d_{n}=d\left(x_{n}, p\right)$ is the error of the nth step. $d(x, y)=\cosh ^{-1}(-\langle x, y\rangle)$ for all $x, y \in \mathbb{H}^{3}$ and $p=(0,0,0,1)$ is the unique fixed 
point. For these algorithms, we are going to consider the sequence of parameters $\alpha_{n}=\beta_{n}=$ $\delta_{n}=0.1+\frac{1}{n+4}$ for each $n=0,1,2, \cdots$.

With a random initial point $x_{0}$ :

$$
x_{0}=(0.69445440978475,1.01382609280137,0.99360871330745,1.87012527625153) \text {, }
$$

the computational results of S-iteration method of rank 2, S-iteration method of rank 3 and Mann iteration method are presented in Table 1 and Fig. 1 for behavior of $d_{n}$. It is observed that the algorithm (4.5) has better performance than other iterative methods.

\begin{tabular}{|l|l|l|l|}
\hline$d_{n}$ & S-iteration of rank 2 $(4.5)$ & S-iteration of rank 3 (4.6) & Mann iteration (4.4) \\
\hline \hline$d_{1}$ & 0.191967402032027 & 0.308695967461188 & 0.371549810384576 \\
\hline \hline$d_{2}$ & 0.003839348040589 & 0.088287046693902 & 0.148619924153828 \\
\hline \hline$d_{3}$ & 0.000290084074068 & 0.028434968816675 & 0.069355964605118 \\
\hline \hline$d_{4}$ & 0.000042506198959 & 0.010060341707828 & 0.035668781796922 \\
\hline \hline$d_{5}$ & 0.000008554382741 & 0.003832675805004 & 0.019617829988310 \\
\hline \hline$d_{6}$ & 0.000002093069529 & 0.001548958314117 & 0.011334746215455 \\
\hline \hline$d_{7}$ & 0.000000585901834 & 0.000656758325161 & 0.006800847729296 \\
\hline \hline$d_{8}$ & 0.000000181280449 & 0.000289712824844 & 0.004204160414466 \\
\hline \hline$d_{9}$ & 0.000000059604644 & 0.000132111730285 & 0.002662634929164 \\
\hline \hline$d_{10}$ & 0.000000021073424 & 0.000061965998202 & 0.001720471800481 \\
\hline \hline$d_{11}$ & 0.000000021073424 & 0.000029776919981 & 0.001130595754731 \\
\hline \hline$d_{12}$ & 0 & 0.000014612744538 & 0.0007537305029357 \\
\hline \hline$d_{13}$ & 0 & 0.000007304275020 & 0.0005087680898053 \\
\hline$T_{A}$ & & &
\end{tabular}

TABLE 1. Numerical comparison of Mann iteration method (4.4), S-iteration method (4.5) of rank 2 and S-iteration method (4.6) of rank 3

\section{ApPLiCATIONS}

5.1. The numerical application to inclusion problems. Let $\mathbb{M}$ be a Hadamard manifold and $\mathscr{X}(\mathbb{M})$ be the set of all set-valued vector fields. Let $V \in \mathscr{X}(\mathbb{M})$ be a set-valued vector field. A point $u \in \mathbb{M}$ is said to be a singularity of $V$ if and only if $\mathbf{0} \in V(u)$. The set of all singularities of $V$ is denoted by

$$
V^{-1}(\mathbf{0})=\{u \in \mathbb{M}: \mathbf{0} \in V(u)\} .
$$

Consider the following inclusion problem in the setting of Hadamard manifolds : Find $u^{*} \in \mathbb{M}$ such that

$$
u^{*} \in V^{-1}(\mathbf{0}) \cap W^{-1}(\mathbf{0}),
$$

where $V, W \in \mathscr{X}(M)$. The solution set of inclusion problem (5.1) is denoted by $\mathbb{S}$.

Thus, the problem of finding singularities of $V$ turns into the problem of finding fixed points of mapping $J_{\mu}^{V}$.

Theorem 5.1. Let $V \in \mathscr{X}(\mathbb{M})$ and $W \in \mathscr{X}(\mathbb{M})$ be two set-valued monotone vector fields on a manifold $\mathbb{M}$ such that $V^{-1}(\mathbf{0}) \cap W^{-1}(\mathbf{0}) \neq \emptyset$. Let $\left\{\alpha_{n}\right\},\left\{\beta_{n}\right\}$ and $\left\{\delta_{n}\right\}$ be sequences of real 


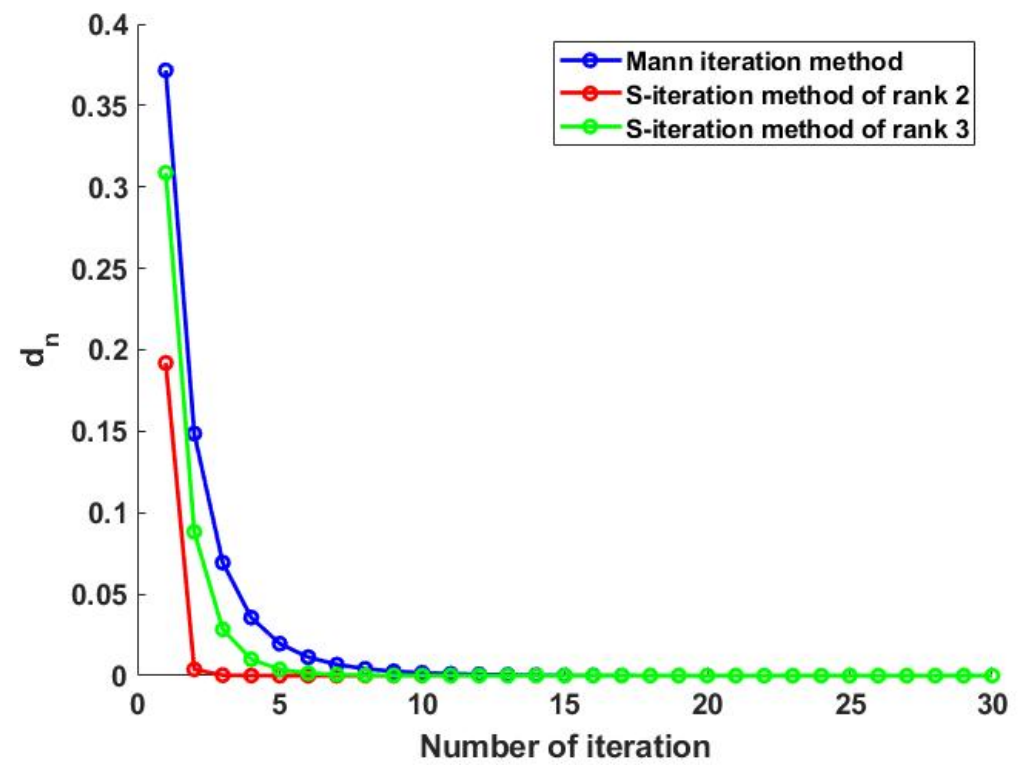

FIGURE 1. Graphical comparison of Mann iteration method (4.4), S-iteration method (4.5) of rank 2 and S-iteration method (4.6) of rank 3

numbers such that $0<\underline{a} \leq \alpha_{n} \leq \bar{a}<1,0<\underline{b} \leq \beta_{n} \leq \bar{b}<1$ and $0<\underline{c} \leq \gamma_{n} \leq \bar{c}<1$, for all $n \in \mathbb{N}_{0}$. Let $x_{0} \in K$ and $\left\{x_{n}\right\}$ be the sequence generated by

$$
\left\{\begin{array}{l}
x_{n+1}=\exp _{J_{\lambda}^{V} x_{n}}\left(1-\alpha_{n}\right) \exp _{J_{\lambda}^{V} x_{n}}^{-1} J_{\mu}^{W}\left(y_{n}\right), \\
y_{n}=\exp _{J_{\lambda}^{V} x_{n}}\left(1-\beta_{n}\right) \exp _{J_{\lambda}^{V} x_{n}}^{-1} J_{\mu}^{W}\left(z_{n}\right), \\
z_{n}=\exp _{x_{n}}\left(1-\delta_{n}\right) \exp _{x_{n}}^{-1} J_{\mu}^{W}\left(x_{n}\right), \quad \forall n \in \mathbb{N}_{0}
\end{array}\right.
$$

where $\lambda, \mu>0$. Then $\left\{x_{n}\right\}$ converges to an element of $V^{-1}(\mathbf{0}) \cap W^{-1}(\mathbf{0})$.

Proof. Set $S=J_{\lambda}^{V}$ and $T=J_{\mu}^{W}$. From Lemma 2.3, $S$ and $T$ are single valued firmly nonexpansive. Hence, they are nonexpansive with $\operatorname{Fix}(S)=V^{-1}(\mathbf{0})$ and $\operatorname{Fix}(T)=W^{-1}(\mathbf{0})$. From the assumption, $\operatorname{Fix}(S) \cap \operatorname{Fix}(T)=V^{-1}(\mathbf{0}) \cap W^{-1}(\mathbf{0}) \neq \emptyset$. Therefore, the desired result follows from Theorem 3.1 immediately.

Example 5.1. Let $\mathbb{R}_{++}=\{x \in \mathbb{R}: x>0\}$ and $\mathbb{M}=\left(\mathbb{R}_{++},\langle\cdot, \cdot\rangle\right)$ be the Riemannian manifold with the Riemannian metric $\langle u, v\rangle=u v$. Then the sectional curvature of $\mathbb{M}$ is zero. The tangent plane at $x \in \mathbb{M}$, denoted by $T_{x} \mathbb{M}$, equals $\mathbb{R}$. The Riemannian distance $d: \mathbb{M} \times \mathbb{M} \rightarrow \mathbb{R}^{+}$is given by

$$
d(x, y)=|\ln (x / y)| \text {. }
$$

Then $\mathbb{M}$ is a Hadamard manifold with sectional curvature 0. For more details, see [5, 7].

Let $\gamma:[0,1] \rightarrow \mathbb{M}$ be a geodesic starting from $x=\gamma(0)$ with velocity $v=\gamma^{\prime}(0) \in T_{x} \mathbb{M}$ defined by

$$
\gamma(t)=x e^{(v / x) t}
$$

Then $\exp _{x}: T_{x} \mathbb{M} \rightarrow \mathbb{M}$ is defined by

$$
\exp _{x} t v=x e^{(v / x) t}
$$


For any $x, y \in \mathbb{M}$, we have

$$
y=\exp _{x}\left(d(x, y) \frac{\exp _{x}^{-1} y}{d(x, y)}\right)=x e^{\left(\exp _{x}^{-1} y\right) / x},
$$

and therefore, the inverse of exponential map is

$$
\exp _{x}^{-1} y=x \ln (y / x)
$$

Let $V: \mathbb{M} \rightrightarrows \mathbb{R}$ be a vector field defined by

$$
V(x)= \begin{cases}0 & \text { if } 0<x<1 \\ t \ln (x): t \in(0, \infty) & \text { if } x \geq 1\end{cases}
$$

Then $V$ is a monotone vector field. For more details, see [5].

Now, note that, for $\mu>0$,

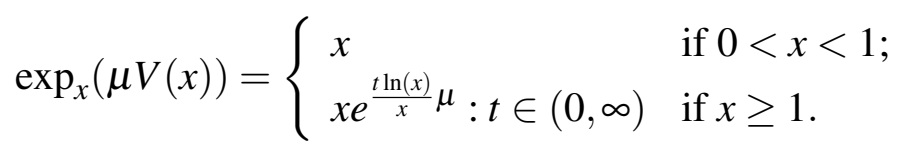

From the definition of the resolvent, we can write

$$
\begin{aligned}
J_{\mu}^{V}(x) & =\left\{z \in \mathbb{M}: x=\exp _{z}(\mu V(z))\right\} \\
& = \begin{cases}x, & \text { if } 0<x<1 ; \\
\left\{z: x=z e^{t \frac{\ln (z)}{z}}\right\} & \text { if } x \geq 1 .\end{cases}
\end{aligned}
$$

Now, we consider two vector fields $V: \mathbb{M} \rightarrow \mathbb{R}, W: \mathbb{M} \rightarrow \mathbb{R}$ defined by

$$
V(x)= \begin{cases}0 & \text { if } 0<x<1 \\ \ln (x) & \text { if } x \geq 1\end{cases}
$$

and

$$
W(x)= \begin{cases}0 & \text { if } 0<x<1 \\ 5 \ln (x) & \text { if } x \geq 1\end{cases}
$$

Then, both $V$ and $W$ are monotone, and $V^{-1}(\mathbf{0})=(0,1]$ and $W^{-1}(\mathbf{0})=(0,1]$. Thus, the common solution set is given by $V^{-1}(\mathbf{0}) \cap W^{-1}(\mathbf{0})=(0,1]$. Let $\lambda, \mu>0$. By the definition of the resolvent, we get

$$
J_{\lambda}^{V}= \begin{cases}x & \text { if } 0<x<1 \\ \left\{z: x=z e^{\frac{\lambda \ln (z)}{z}}\right\} & \text { if } x \geq 1\end{cases}
$$

and

$$
J_{\mu}^{W}= \begin{cases}x & \text { if } 0<x<1 \\ \left\{z: x=z e^{\frac{5 \mu \ln (z)}{z}}\right\} & \text { if } x \geq 1\end{cases}
$$

From Theorem 5.1, the sequence generated by equation (5.1) converges to the solution of problem $V^{-1}(\mathbf{0}) \cap W^{-1}(\mathbf{0})$. 
5.2. Convex feasibility problems. Let $C$ and $D$ be nonempty, closed, and geodesic convex subsets of a Hadamard manifold $\mathbb{M}$ such that $C \cap D \neq \emptyset$. Since $P_{C}: \mathbb{M} \rightarrow C$ with $F$ ix $\left(P_{C}\right)=C$ and $P_{D}: \mathbb{M} \rightarrow D$ with $\operatorname{Fix}\left(P_{D}\right)=D$ are nonexpansive. Therefore, $F i x\left(P_{C}, P_{D}\right)=C \cap D$. Thus, finding a point in the intersection of two nonempty, closed, and geodesic convex subsets of a Hadamard manifold is equivalent to finding an element in $\operatorname{Fix}\left(P_{C}, P_{D}\right)$.

Finally, we apply Algorithm 3.2 to find a point in the intersection of two nonempty, closed, and geodesic convex subsets of a Hadamard manifold. The following theorem gives criteria for finding the common element of two convex set, which is an extension of Theorem 7 of [17] from Hilbert spaces to manifolds.

Theorem 5.2. Let $C$ and $D$ be nonempty, closed and geodesic convex subset of a Hadamard manifold $\mathbb{M}$ such that $C \cap D \neq \emptyset$. Let $\left\{\alpha_{n}\right\},\left\{\beta_{n}\right\}$ and $\left\{\delta_{n}\right\}$ be sequences of real numbers such that $0<\underline{a} \leq \alpha_{n} \leq \bar{a}<1,0<\underline{b} \leq \beta_{n} \leq \bar{b}<1$ and $0<\underline{c} \leq \gamma_{n} \leq \bar{c}<1$, for all $n \in \mathbb{N}_{0}$. Let $x_{0} \in K$ and $\left\{x_{n}\right\}$ be the sequence generated by

$$
\left\{\begin{array}{l}
x_{n+1}=\exp _{P_{C}\left(x_{n}\right)}\left(1-\alpha_{n}\right) \exp _{P_{C}\left(x_{n}\right)}^{-1} P_{D}\left(y_{n}\right), \\
y_{n}=\exp _{P_{C}\left(x_{n}\right)}\left(1-\beta_{n}\right) \exp _{P_{C}\left(x_{n}\right)}^{-1} P_{D}\left(z_{n}\right), \\
z_{n}=\exp _{x_{n}}\left(1-\delta_{n}\right) \exp _{x_{n}}^{-1} P_{D}\left(x_{n}\right), \quad \forall n \in \mathbb{N}_{0} .
\end{array}\right.
$$

Then $\left\{x_{n}\right\}$ converges to an element of $C \cap D$.

Proof. Set $S=P_{C}$ and $T=P_{D}$. Then $S, T: \mathbb{M} \rightarrow \mathbb{M}$ are nonexpansive with $\operatorname{Fix}(S)=C$ and $\operatorname{Fix}(T)=D$. From the assumption, $\operatorname{Fix}(S) \cap F i x(T)=C \cap D \neq \emptyset$. Therefore, the desired result follows from Theorem 3.1 immediately.

\section{Acknowledgment}

The authors are grateful to the referees for useful suggestions which improved the contents of this paper.

\section{REFERENCES}

[1] R.P. Agarwal, D. O'Regan, D.R. Sahu, Fixed point theory for Lipschitzian-type mappings with applications, Springer, New York, 2009.

[2] R.P. Agarwal, D. O'Regan, D.R. Sahu, Iterative construction of fixed points of nearly asymptotically nonexpansive mappings, J. Nonlinear Convex Anal. 8 (2007), 61-79.

[3] S. Al-Homidan, Q.H. Ansari, F. Babu, Halpern and Mann type algorithms for fixed points and inclusion problems on Hadamard manifolds, Numer. Funct. Anal. Optim. 40 (2019), 621-653.

[4] S. Al-Homidan, Q.H. Ansari, F. Babu, J.C. Yao, Viscosity method with a $\phi$-contraction mapping for hierarchical variational inequalities on Hadamard manifolds, Fixed Point Theory, 21 (2020), 561-584.

[5] Q.H. Ansari, F. Babu, J.C. Yao, Regularization of proximal point algorithms in Hadamard manifolds, J. Fixed Point Theory Appl. 21 (2019), Article ID 25.

[6] M. Bridson, A. Haefliger, Metric Spaces of Non-positive Curvature, Springer- Verlag, Berlin, Heidelberg, New York, 1999.

[7] J.X. Da Cruz Neto, O.P. Ferreira, L.R. Lucambio Pérez, S.Z. Németh, Convex and monotone-transformable mathematical programming problems and a proximal-like point methodJ. Global Optim. 35 (2006), 53-69.

[8] M. P. Do Carmo, Riemannian Geometry, Birkha̋user, Boston, 1992.

[9] W.R. Mann, Mean value methods in iterations, Proc. Amer. Math. Soc. 4 (1953), 506-510.

[10] J. Fan, X. Qin, Tseng's extragradient algorithm for pseudomonotone variational inequalities on Hadamard manifolds. Appl. Anal. (2020), DOI: 10.1080/00036811.2020.1807012. 
[11] B. Halpern, Fixed points of non-expansive maps, Bull. Amer. Math. Soc. 73 (1967), 591-597.

[12] T. H. Kim, H. K. Xu, Strong convergence of modified Mann iterations, Nonlinear Anal. 61 (2005), 51-60.

[13] C. Li, G. López, V. Martín-Márquez, Iterative algorithms for nonexpansive mappings in Hadamard manifolds, Taiwanese J. Math. 14 (2010), 541-559.

[14] C. Li, G. López, V. Martín-Márquez, Monotone vector fields and the proximal point algorithm on Hadamard manifolds, J. Lond. Math. Soc. 79 (2009), 663-683

[15] C. Li, G. López, V. Martín-Márquez, J.H. Wang, Resolvents of set valued monotone vector fields in Hadamard manifolds, Set-Valued Var. Anal. 19 (2011), 361-383

[16] S. Riech, Weak convergence theorems for non-expansive mappings in Banach spaces, J. Math. Anal. Appl. 67 (1979), 274-276.

[17] D. R. Sahu, A. Pitea, M. Verma, A new iteration technique for nonlinear operators as concerns convex programming and feasibility problems, Numer. Algo. 83 (2020), 421-449.

[18] D. R. Sahu, Applications of accelerated computational methods for quasi-nonexpansive operators to optimization problems, Soft Comput. 24 (2020), 17887-17911.

[19] D. R. Sahu, J. C. Yao, M. Verma, K. K. Shukla, Convergence rate analysis of proximal gradient methods with applications to composite minimization problems, Optimization, (2020), 10.1080/02331934.2019.1702040.

[20] D. R. Sahu, Applications of the S-iteration process to constrained minimization problems and split feasibility problems, Fixed Point Theory 12 (2011), 187-204.

[21] T. Sakai, Riemannian Geometry, Translations of Mathematical Monographs, American Mathematical Society, Providence 149, 1996.

[22] C. Udriste, Convex functions and optimization methods on Riemannian manifolds, Mathematics and its Applications 297, Kluwer, Dordrecht, 1994. 